\title{
Defective nuclear entry of hydrolases prevents neutrophil extracellular trap formation in patients with chronic granulomatous disease
}

Romao, Susana ; Puente, Emilio Tejera ; Nytko, Katarzyna J ; Siler, Ulrich ; Münz, Christian ; Reichenbach, Janine

DOI: https://doi.org/10.1016/j.jaci.2015.09.007

Posted at the Zurich Open Repository and Archive, University of Zurich ZORA URL: https://doi.org/10.5167/uzh-118131

Journal Article

Accepted Version

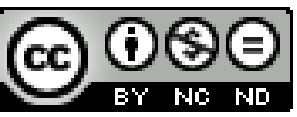

The following work is licensed under a Creative Commons: Attribution-NonCommercial-NoDerivatives 4.0 International (CC BY-NC-ND 4.0) License.

Originally published at:

Romao, Susana; Puente, Emilio Tejera; Nytko, Katarzyna J; Siler, Ulrich; Münz, Christian; Reichenbach, Janine (2015). Defective nuclear entry of hydrolases prevents neutrophil extracellular trap formation in patients with chronic granulomatous disease. Journal of Allergy and Clinical Immunology, 136(6):17031706.e5.

DOI: https://doi.org/10.1016/j.jaci.2015.09.007 
Romao et al., JACI-D-15-00119

3 Dr. Susana Romao ${ }^{1,2}$, Dr. Emilio Tejera Puente ${ }^{2,3}$, Dr. Katarzyna J. Nytko ${ }^{2,4}$, Dr. Ulrich Siler ${ }^{2}$, 4 Prof. Christian Münz ${ }^{1}$ and Prof. Janine Reichenbach ${ }^{2}$

5

$6{ }^{1}$ Viral Immunobiology, Institute of Experimental Immunology, University of Zurich, Switzerland

$7 \quad 2$ Division of Immunology, University Children's Hospital Zurich and Children's Research

8 Center, Switzerland

$9{ }^{3}$ Current affiliation: Molecular, Cellular and Developmental Neurobiology, Instituto Cajal,

10 CSIC, Madrid, Spain

$11{ }^{4}$ Current affiliation: Laboratory for Molecular Radiobiology, Department of Radiation Oncology,

12 University Hospital Zürich

14 Corresponding author:

15 Prof. Janine Reichenbach, University Children's Hospital Zürich, Div. of Immunology, 16 Steinwiesstr. 75, CH-8032 Zürich, Phone: + 41 442667311, Fax: +41 442667986, 17 janine.reichenbach@kispi.uzh.ch 
Romao et al., JACI-D-15-00119

\section{Capsule summary}

20 Hydrolases perinuclearly accumulate in activated neutrophils of CGD patients or after inhibition

21 of PI3-kinase dependent ROS production, preventing their nuclear access and the resulting

22 NETosis. Pharmacologically bypassing this bottleneck might restore antimicrobial defenses in

23 CGD.

24

25 Keywords

26 neutrophil elastase, PI3-kinase, NADPH oxidase, NETosis, macroautophagy, chronic 27 granulomatous disease, CGD

29 Abbreviations

$30 \mathrm{CGD}=$ chronic granulomatous disease, $\mathrm{HD}=$ healthy donors, $3-\mathrm{MA}=3$-methyladenine, $\mathrm{NE}=$ 31 neutrophil elastase, NET neutrophil extracellular trap, NETosis = NET formation, NOX2 =

32 phagocytic NADPH oxidase, PI3 = phosphoinositide- $3=$ phosphatidylinositol-3, $\mathrm{PMA}=$ Phorbol

33 12-myristate 13-acetate, $\mathrm{ROS}$ reactive oxygen species, $\mathrm{SP}=$ spautin-1. 


\section{To the Editor,}

Neutrophils antimicrobial activity depends on phagocytosis, neutrophil extracellular trap

37 (NET) formation (NETosis), and cytokine production. NETosis is dependent on NADPH oxidase

38 (NOX2) driven ROS production, nuclear access of neutrophil elastase (NE), and histone

39 degradation ${ }^{1}$. ROS production is deficient in patients with chronic granulomatous disease (CGD),

40 resulting in impaired NETosis and recurrent severe bacterial and fungal infection ${ }^{2}$.

41 In order to shed light on the poorly understood steps downstream of ROS, resulting in

42 NETosis, several groups have recently explored macroautophagy ${ }^{3-8}$, a cellular degradation

43 pathway for cytoplasmic constituent delivery to lysosomes, which reshapes membrane

44 compartments and could, therefore, be involved in hydrolase access to the nucleus. However, in

45 all of these studies no direct correlation of macroautophagic activity and NETosis could be

46 reported, and only pharmacological inhibition of phosphatidylinositol-3 (PI3)-kinase activity was

47 used to compromise macroautophagy. Therefore, we decided to revisit the regulation of

48 macroautophagy and ROS production by PI3-kinase inhibition during NETosis in CGD and

49 healthy donor (HD) neutrophils.

50 NETosis was induced by PMA (Fig 1, A) or Candida albicans (Fig 1, B) stimulation

51 together with PI3-kinase inhibitors in human HD and CGD neutrophils, and DNA release was

52 followed over time by SYTOX assays. Both stimuli resulted in NETosis in HD neutrophils.

53 However, only inhibition of PI3-kinases through 3-methyladenine (3-MA) caused a significant

54 decrease in NETosis induced by both stimuli. Previously, nuclear NE translocation was suggested

55 to initiate chromatin decondensation and subsequent NETosis ${ }^{1}$. NE's nuclear translocation, as

56 assessed by confocal microscopy, was also reduced with 3-MA after opsonized yeast and PMA

57 incubation (Fig E1, A). Therefore, the PI3-kinase inhibitor 3-MA, but to a lesser extent other PI3-

58 kinase inhibitors, like wortmannin and spautin-1, can inhibit NETosis. 
Since 3-MA is classically used as a macroautophagy inhibitor, we investigated accumulation of autophagosomes in neutrophils treated with opsonized yeast and PMA $\pm 3-\mathrm{MA}$ through immunofluorescent quantification of dots positive for LC3B, which gets attached to autophagosome membranes. Interestingly, upon 1h stimulation, 3-MA did not seem to effectively reduce autophagosome formation when compared to untreated cells (Fig 1, C). In addition, membrane association of LC3B, detected as LC3-II levels by Western blotting, was not 65 significantly different \pm 3-MA (Fig E1, B). Along these lines, neutrophils of NETosisincompetent CGD patients induced macroautophagy to similar extent as HDs after stimulation with opsonized yeast or PMA (Fig E2). In contrast, PI3-kinase inhibition with 3-MA consistently reduced both yeast- and PMA-induced ROS production (Fig 1, D), suggesting that the negative effect of 3-MA on NETosis might be related to the ineffectiveness of cells to generate ROS rather than blocking macroautophagy. Together, these results point to a macroautophagy-independent, but ROS dependent mechanism of PI3-kinase to control NETosis in human neutrophils.

To further investigate the steps leading to NETosis, we looked into the kinetics of NE

73 localization. Confocal microscopy of NE in PMA-treated HD and CGD neutrophils confirmed 74 the absence of extracellular or intra-nuclear NE staining in CGD neutrophils (Fig 2, A and B). 1h 75 after PMA-stimulation, CGD and HD cells recruited NE to the perinuclear region. If this NE is 76 granula-bound or released into the cytosol and physically associated with the nuclear membrane 77 could not be determined. 3h after PMA-treatment, NE was still accumulating around CGD cell 78 nuclei, whereas in HD NE had already migrated into the nuclear region. Similarly, 3-MA treated 79 cells showed a trend towards stabilization of NE staining in the perinuclear area $3 \mathrm{~h}$ post PMA80 treatment (Fig 1, E; Fig E3). As a second measure of neutrophil hydrolases' access to the 81 nucleus, we assessed proteolysis of histone H4 (Fig 2, C; Fig E4). After PMA-activation, HD 82 neutrophils initiated NETosis and H4 was degraded to allow DNA decondensation (Fig E4, A), 
83 whereas in CGD cells, H4 levels remained stable $3 \mathrm{~h}$ and $4 \mathrm{~h}$ after PMA-induction (Fig E4, B).

84 This indicates that neutrophils deficient in phagosome-associated NOX2-mediated ROS 85 production are unable to induce NE nuclear translocation, but can nevertheless accumulate NE 86 around their nuclei.

87 To confirm that the classical macroautophagy machinery was not involved in the transport 88 of vesicular NE to the nucleus, we assessed perinuclear co-localization of LC3B and NE in 89 neutrophils isolated from HDs by confocal microscopy. As previously observed (Fig 2), NE 90 translocated to the area around the nucleus 1h post-stimulation with PMA (Fig E1, C and D), 91 while LC3B did not accumulate there. These results indicate that NE trafficking to the nucleus 92 does not depend on autophagosomes.

In contrast, previous studies had suggested that PI3-kinase inhibition affects NETosis via 94 compromising macroautophagy ${ }^{3-8}$. Most of these studies, however, did not analyse the effect of 95 PI3-kinase inhibition on ROS production ${ }^{4-6,8}$. Moreover, while PI3-kinase inhibition with 3-MA 96 was reliably able to down-modulate DNA-release after NETosis stimulation, autophagosome97 associated LC3-II levels could not be decreased in some of these studies, and vice versa the PI398 kinase inhibitor wortmannin was able to compromise macroautophagy in some studies, but did 99 not affect DNA-release $3,4,7,8$. In addition, macroautophagy stimulation by inhibition of the 100 mammalian target of rapamycin (mTOR) increased DNA-release after NETosis stimulation, but 101 it was not analyzed if macroautophagy inhibition would block this synergistic effect ${ }^{6}$. Thus, these 102 previous studies and ours demonstrate that 3-MA reliably diminishes DNA-release during 103 NETosis, but that LC3-II coupled autophagic membranes are not decreased in most studies ${ }^{7}$, 104 while 3-MA compromises NOX2-dependent ROS formation. and function of many proteins in the cell. These effector proteins usually contain the lipid- 
Romao et al., JACI-D-15-00119

107 binding domains FYVE or PX. For example, the PX domain of NOX2 p40phox subunit was 108 described to be important for effective ROS production upon phagocytosis of opsonized-

109 bacteria 9 . Accordingly, PI3-kinase inhibition in neutrophils may prevent correct assembly of 110 NOX2 and therefore diminish the oxidative burst in these cells, affecting their NETotic response. 111 Indeed, we observed diminished ROS production upon 3-MA treatment. Thus, we favor the 112 hypothesis that PI3-kinase inhibition compromises NETosis by inhibiting ROS formation to 113 prevent neutrophil hydrolase access to the nucleus for DNA decondensation. If ROS formation 114 represents the central axis for triggering NETosis in neutrophils, interfering with this pathway 115 should be harnessed clinically.

\section{Acknowledgements}

118 We are grateful to all CGD patients, relatives, and blood donors for their participation in this 119 study, Prof. Arturo Zychlinsky for providing the anti-NE antibody and for fruitful and 120 constructive discussions. 
Romao et al., JACI-D-15-00119

122

123 1. Papayannopoulos V, Metzler KD, Hakkim A, Zychlinsky A. Neutrophil elastase and

\section{References} myeloperoxidase regulate the formation of neutrophil extracellular traps. J Cell Biol 2010; 191:677-91.

2. Bianchi M, Hakkim A, Brinkmann V, Siler U, Seger RA, Zychlinsky A, et al. Restoration of NET formation by gene therapy in CGD controls aspergillosis. Blood 2009; 114:261922.

3. Remijsen Q, Vanden Berghe T, Wirawan E, Asselbergh B, Parthoens E, De Rycke R, et al. Neutrophil extracellular trap cell death requires both autophagy and superoxide generation. Cell Res 2011; 21:290-304.

4. Cheng ML, Ho HY, Lin HY, Lai YC, Chiu DT. Effective NET formation in neutrophils from individuals with G6PD Taiwan-Hakka is associated with enhanced NADP ${ }^{+}$ biosynthesis. Free Radic Res 2013; 47:699-709.

5. Mitroulis I, Kambas K, Chrysanthopoulou A, Skendros P, Apostolidou E, Kourtzelis I, et al. Neutrophil extracellular trap formation is associated with IL-1beta and autophagyrelated signaling in gout. PLoS ONE 2011; 6:e29318.

6. Itakura A, McCarty OJ. Pivotal role for the mTOR pathway in the formation of neutrophil extracellular traps via regulation of autophagy. Am J Physiol Cell Physiol 2013; 305:C348-54.

7. Maugeri N, Campana L, Gavina M, Covino C, De Metrio M, Panciroli C, et al. Activated platelets present high mobility group box 1 to neutrophils, inducing autophagy and promoting the extrusion of neutrophil extracellular traps. J Thromb Haemost 2014; $12: 2074-88$. 
Romao et al., JACI-D-15-00119

145 8. Tang S, Zhang Y, Yin S, Gao X, Shi W, Wang Y, et al. Neutrophil extracellular trap 146 formation is associated with autophagy-related signaling in ANCA-associated vasculitis. $147 \quad$ Clin Exp Immunol 2015.

148 9. Ellson C, Davidson K, Anderson K, Stephens LR, Hawkins PT. PtdIns3P binding to the 149 PX domain of p40phox is a physiological signal in NADPH oxidase activation. Embo $\mathbf{J}$ 150 $2006 ; 25: 4468-78$.

151

152

153 
Romao et al., JACI-D-15-00119

\section{Funding}

155 This work was supported by Cancer Research Switzerland (KFS-02652-08-2010 and KFS-3234156 08-2013), Worldwide Cancer Research (11-0516 and 14-1033), $\mathrm{KFSP}^{\mathrm{MS}}$ and KFSP ${ }^{\mathrm{HHLD}}$ of the 157 University of Zurich, the Baugarten Foundation, the Sobek Foundation, Fondation Acteria, the 158 Swiss Vaccine Research Institute, EU-FP7 COST program "Mye-EUNITER" and the Swiss 159 National Science Foundation (310030_143979 and CRSII3_136241) to CM, and by the Gebert 160 Rüf Stiftung program "Rare Diseases - New Approaches” (grant no. GRS-046/10), EU-FP7 161 CELL-PID HEALTH-2010-261387 and EU-FP7 NET4CGD, Zurich Centre for Integrative 162 Human Physiology (ZIHP), Gottfried und Julia Bangerter-Rhyner-Stiftung to JR, and Fondazione 163 Ettore e Valeria Rossi to US and JR.. 


\section{Figure Legends}

\section{Figure 1}

PI3-kinase inhibition decreases NETosis and ROS production without altering macroautophagy. Sytox-based NET assay in PMA (A) or C. albicans (B) stimulated neutrophils \pm PI3K-inhibitors $(\mathrm{N}=3$, mean $\pm \mathrm{SD})$. (C) Quantification of macroautophagy in HD neutrophils

171 after 1 h stimulation $(\mathrm{N}=6$, mean $\pm \mathrm{SD})$. (D) ROS production after 30min incubation as indicated 172 (mean \pm SD; ***: $\mathrm{P}<0.001)$. (E) Quantification of NE-signal around nucleus of PMA-stimulated 173 neutrophils +/-3-MA (mean \pm SD).

Figure 2

176 NE nuclear entry requires ROS for histone H4 degradation during NETosis. (A) PMAstimulated HD or CGD neutrophils were analyzed for NE (red; black/white) and DAPI (blue) by immunefluorescence-microscopy (B). NE-signal around the nucleus of PMA-stimulated neutrophils (means $\pm \mathrm{SD}$; **: t-test $\mathrm{P}<0.01$ ). (C) Quantification of fold decrease in $\mathrm{H} 4$ levels in 9 HD and 6 CGD patients neutrophils +/- PMA.

\section{Supplemental material:}

183 Supplemental Figure E1: PI3-kinase inhibition blocks NE release, but minimally affects 184 macroautophagy in human activated neutrophils. (A) Quantification of extracellular or nuclear NE in neutrophils \pm 3 -MA, wortmannin and spautin-1, \pm stimulation with opsonized $C$. albicans (1:1 ratio) or PMA for $3 \mathrm{~h}$ (Pooled data from $4 \mathrm{HD} ; 75$ cells/donor; means \pm SD). HD neutrophils were treated \pm 3-MA and \pm PMA, and LC3-II levels were assessed by Western blot (one blot of at least 3). Numbers below the blot represent the fold increase of LC3-II 189 compared with the non-stimulated control cells after normalization to the corresponding actin 
levels. (C) HD neutrophils were incubated with PMA for $10 \mathrm{~min}, 1 \mathrm{~h}$ or $3 \mathrm{~h}$, fixed, permeabilized 191 and stained for ATG8/LC3 (green), NE (red) and DAPI (blue). Representative confocal pictures 192 of 1 cell out of 50 analyzed are shown. Data from 3 HD. (D) Quantification of fluorescence 193 intensity for ATG8/LC3 and NE signals in the perinuclear region of HD neutrophils $(\mathrm{n}=50)$. 194 Cells were left unstimulated or treated with PMA at the indicated time points. Pooled data from 3 195 HD.

\section{Supplemental Figure E2.}

\section{Intact macroautophagy in CGD neutrophils}

199 Quantification of ATG8/LC3 ${ }^{+}$dots (ATG8/LC3 green, DAPI blue) on neutrophils (n $\geq 100$; 200 means \pm SD) from 3 HD and 4 CGD patients after 1 h stimulation (Scale bars $5 \mu \mathrm{m}$ ).

\section{Supplemental Figure E3.}

203 PI3-kinase inhibition prevents NE access to the nucleus of neutrophils. (A) 204 Immunofluorescence analysis of PMA-stimulated HD neutrophils ( $\mathrm{n}=4$ HD) +/- 3-MA, NE (red: 205 merged color signals, black/white: confocal pictures) and DAPI (blue). Quantification is shown 206 in Fig 1, E.

208 Supplemental Figure E4.

209 ROS triggers nuclear histone degradation. Western blot analysis ( 1 of 6 blots) of histone 4 210 (H4) in HD (A) or CGD neutrophils (B) +/- PMA. Numbers indicate the fold increase of H4 211 compared to non-stimulated control (normalized to actin). Quantification is shown in Fig 2, C. 
A

0 min.

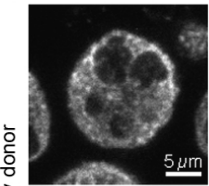

站

O
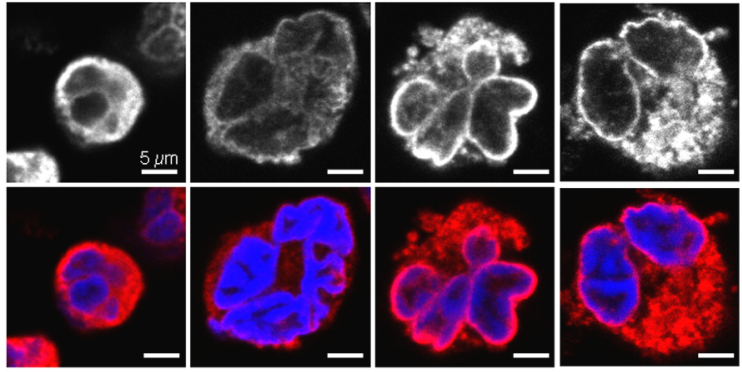

B

\section{茨}
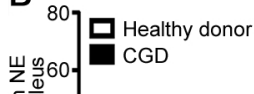

焉 0 c

$$
\circ
$$




\section{Materials and Methods}

\section{Antibodies and cell dyes}

For Western blotting, antibody anti-ATG8/LC3 (clone 5F10) was purchased from Nanotools and antibody anti-histone H4 (clone 62-141-13) was obtained from Millipore. Directly labeled antibeta actin (HRP) antibody was obtained from Abcam. For immunofluorescence stainings antiATG8/LC3 was received from MBL and anti-neutrophil elastase (NE) was kindly provided by Arturo Zychlinsky's lab (Berlin, Germany). Secondary antibodies conjugated to Alexa488 or Alexa555 were purchased from Invitrogen. The nucleic acid stains Sytox ${ }^{\circledR}$ green and DAPI and the redox sensitive probe Amplex ${ }^{\circledR}$ UltraRed were provided by Invitrogen.

\section{Cell preparation}

Blood was drawn from healthy donors (HD) (Zurich_Blood_Center) and 6 CGD patients (University Children's Hospital Zurich) after obtaining informed consent from patients or parents in accordance with the Declaration of Helsinki and local ethical provisions. Neutrophils were isolated by density-gradient centrifugation on Ficoll/Hypaque. The lower layer containing granulocytes was subjected to hypotonic lysis of red blood cells, followed by ice-cold PBS washing. Cells were resuspended in RPMI 1640 medium supplemented with 5\% heat-inactivated fetal bovine serum and used immediately after isolation. For testing the production of reactive oxygen species, cells were kept in PBS throughout the assay.

\section{NET induction}

Neutrophils were left unstimulated or treated with $100 \mathrm{nM}$ phorbol 12-myristate 13-acetate (PMA) or opsonized Candida albicans yeast. C. albicans yeast-locked mutant strain $\Delta$ hgc1 was 
grown overnight at $30^{\circ} \mathrm{C}$ in Sabouraud medium and subcultured to reach the exponential growth phase. For opsonization, $2 \times 10^{7}$ yeast cells were washed in PBS, resuspended in $1 \mathrm{ml}$ of $20 \%$ human plasma (isolated from the top layer after Ficoll/Hypaque gradient centrifugation) and incubated at $37^{\circ} \mathrm{C}$ for $20 \mathrm{~min}$. Cells were again washed in PBS and added to the respective neutrophils at a 1:1 ratio. Where indicated, $30 \mathrm{~min}$ previously previous to 100nM PMA or opsonized $C$. albicans stimulation, cells were treated with the following PI3K inhibitors: $5 \mathrm{mM}$ 3-MA, $50 \mathrm{nM}$ wortmannin or $1 \mu \mathrm{M}$ spautin-1.

NETosis occurred within 2 to $3 \mathrm{~h}$ after stimulation. To quantify the kinetics of NET formation, $5 \times 10^{4}$ neutrophils were seeded per well in flat-bottom 96-well plates, incubated with the appropriate stimuli and $1 \mu \mathrm{M}$ Sytox ${ }^{\circledR}$ green. Fluorescence values were measured by a Tecan Infinite M200 Pro fluorometer (Ex. $485 \mathrm{~nm} / \mathrm{Em} .520 \mathrm{~nm}$ at $37^{\circ} \mathrm{C}$ ) every $20 \mathrm{~min}$ for a total of 280 min. (means $\pm \mathrm{SD} ; 3$ independent assays with different donors). In addition, NETosis was assessed by quantification of the number of cells with positive nuclear staining for NE. Cells ( $\mathrm{n} \geq$ 100/well) were counted from confocal pictures using ImageJ software. Figure 1E: Means \pm Stdev; pooled from 4 HD. Figure 2A: $2 \mathrm{HD}$ and 3 CGD patients; scale bars $=5 \mu \mathrm{m}$.

\section{Immunofluorescence and microscopy}

For immunofluorescence stainings, $4 \times 10^{5}$ neutrophils were seeded per well on poly-lysine treated 8-well chamber slides (Ibidi) and stimulated as indicated for 1h. After treatment, cells were fixed in $3 \%$ paraformaldehyde for $20 \mathrm{~min}$ at $4^{\circ} \mathrm{C}$, permeabilized with $0.5 \%$ Triton-X100 for $1 \mathrm{~min}$ at room temperature, then incubate with the Image-iT FX signal enhancer (Invitrogen) and stained with the indicated antibodies followed by the appropriate secondary reagent. All washes were performed in PBS supplemented with $1 \%$ fish skin gelatin and $0.02 \%$ saponin. Slides were counterstained with DAPI and mounted with 50\% glycerol in PBS. Cells were visualized through 
a x63 1.4 NA oil immersion lens with an inverted CLSM Leica SP5 confocal microscope. For quantification of the fluorescence signal of the different antibodies, part of the perinuclear region was selected to calculate the intensity values using ImageJ software. Data are expressed as dot plots with median value displayed as a horizontal red line. Figure_1, C: Neutrophils of 6 HD; $n \geq$ 100 cells per well; scale bars $=5 \mu \mathrm{m}$.

\section{Measurement of reactive oxygen species}

Production of reactive oxygen species by neutrophils was assessed with the cell-permeable fluorescent probe Amplex ${ }^{\circledR}$ UltraRed. Neutrophils were seeded in flat-bottom 96-well plates, $2 \times 10^{5}$ cells/well, stimulated with the opsonized $C$. albicans (1:1 ratio) or 100nM PMA and with a mixture of $25 \mu \mathrm{M}$ of Amplex ${ }^{\circledR}$ UltraRed and $0.5 \mathrm{U} / \mathrm{ml} \mathrm{HRP}$ for $30 \mathrm{~min}$ at $37^{\circ} \mathrm{C}$. Fluorescence values were analyzed in a Tecan Infinite M200 Pro fluorometer (Ex. 530 nm / Em. 580 nm). Figure 1, C: Triplicates from 2 independent HD

\section{Western blot analysis}

To obtain protein extracts, at the indicated times, 6x Laemmli buffer with $1 \% \beta$-mercaptoethanol was added to the neutrophils at a final concentration of 1x. Cells were scrapped off the wells and sonicated for $30 \mathrm{sec}$ at $50 \%$ intensity. Resulting cell lysates were frozen at $-80^{\circ} \mathrm{C}$ for later immunoblotting. For Western blot analysis, cell lysates were boiled for 5 min, resolved by SDSPAGE and transferred onto Polyvinylidenfluorid membranes. For detection of primary antibodies, HRP-conjugated secondary antibodies and the ECL femto detection system were used. Membranes were visualized in a Vilber Lourmat Fusion FX imaging system or exposed to films and densitometry was performed using the ImageJ software. 


\section{Histone degradation assay}

Neutrophils were seeded at a density of $5 \times 10^{5}$ cells/well in 48-well plates. After stimulation with PMA for the indicated times, cell lysates were generated and H4 degradation was analyzed by immunoblotting.

\section{Statistical analysis}

Where indicated, unpaired student's t-tests were performed using the GraphPad Prism Software (Version 5.0a). 


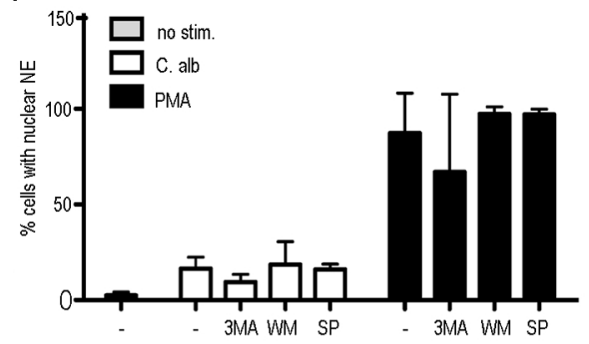

B

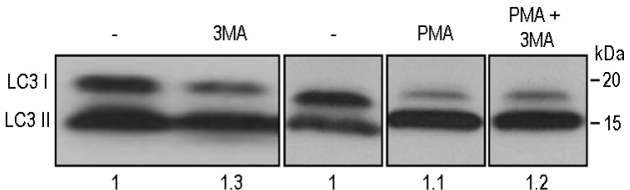

C
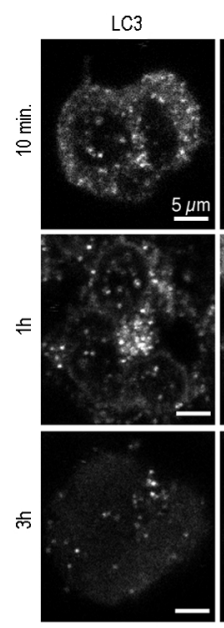

D
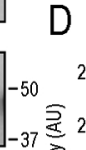

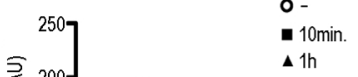

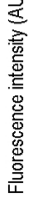

× $1 \mathrm{~h}$
NE
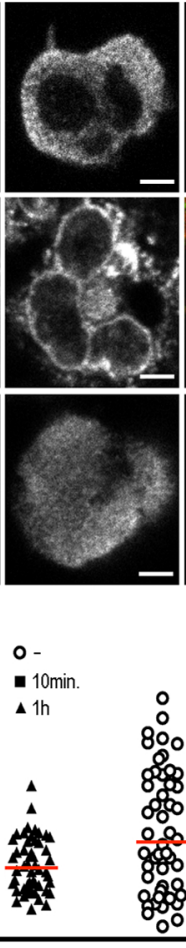

Perinuclear LC3

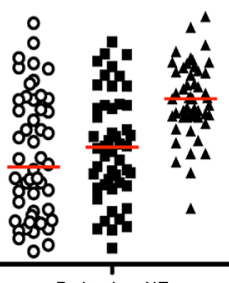

Perinuclear NE

Supplementary Figure E1 


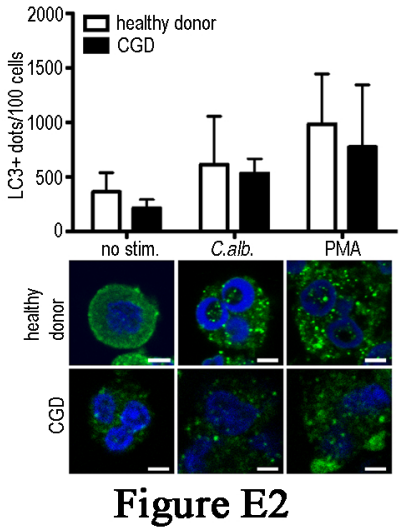



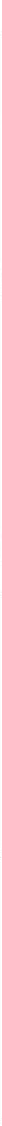

Figure E3 
$A$

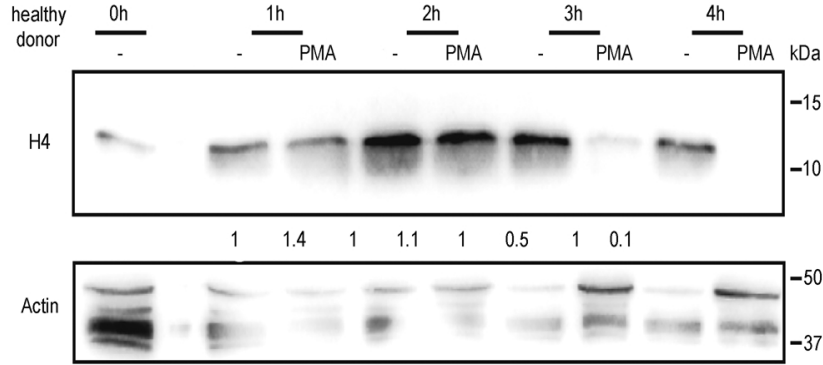

B

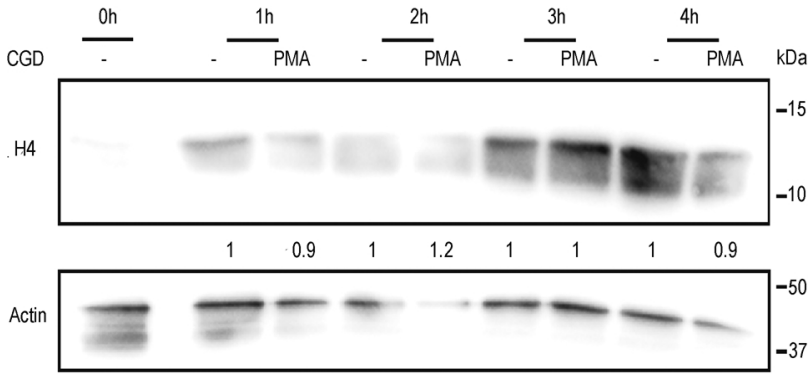

Figure E4 